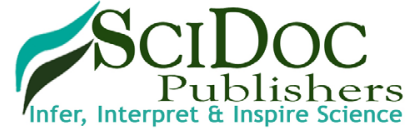

International Journal of Forensic Science \& Pathology (IJFP) ISSN 2332-287X

\title{
Ethical Issues Surrounding the Research Use of Forensic Samples in Japan
}

Research Article

Tatsuaki Tsuruyama*, Hikari Inoue

Center for Anatomical, Pathological, and Forensic Medical Research, Graduate School of Medicine, Kyoto University, Yoshida-Konoe-cho, Sakyo-ku, Kyoto, Japan.

\section{Abstract}

In recent years, many countries have established ethical legislation to regulate the use of human samples in medical research. In this paper, we consider the unique ethical issues involved when forensic samples are used as research resources. Unlike clinical pathological autopsies that presuppose the informed consent of a patient's relative(s) for conduct of the autopsy, certain aspects of forensic autopsies does not. In this article, we classify the forensic research and consider the ethical issues in individual research use and propose criteria for research use based on scientific validation and an examination by an institutional ethical review board.

\section{Background}

\section{Informed Consent in Forensic Autopsies}

The current authors previously reported on ethical guidelines in Japan for the use of human samples for research purposes [1, 2]. In Japan, Ethical Guidelines for Medical and Health Research Involving Human Subjects (EGM) have been established primarily by the Ministry of Education, Culture, Sports, Science and Technology and the Ministry of Health, Labour and Welfare, and applied research in which human samples are used [3]. The guidelines state that the donor's informed consent (IC) is necessary in order to use donor samples for research purposes. The guidelines are primarily referring to the use of surgical samples or biopsy and blood samples collected during a clinical examination. Ethical issues surrounding the research use of these samples are comparatively limited because IC is obtained from donors. For example, lack of family consent is a major reason for not performing an autopsy of persons with epilepsy [4]. In contrast, the research use of samples obtained through forensic autopsies has been considered from ethical views because IC may not necessarily be obtained from the relatives. Although the Guidelines of the Japanese Association of Forensic Medicine recommend that forensic pathologists obtain IC from patient's relatives prior to the storage and use of autopsy samples, it can be difficult to make contact with them for obtaining during an inquest procedure [5]. At present, the institutes that govern forensic autopsies and coroner inquests differ among various countries and it is thus difficult to discuss from a comprehensive perspective. In this paper, we will first consider the situation in Japan and then proceed to a discussion of the use of forensic samples in order to highlight the general issues surrounding using these samples in research.

\section{Forensic Autopsies in Japan (Administrative and Judicial)}

In Japan, a forensic autopsy is divided into two categories: administrative and judicial. The former is conducted according to the Autopsy Preservation Act, while the latter is conducted according to the Code of Criminal Procedure.

After a physician acknowledges an unnatural or suspicious death, he or she files a report and notifies the police, pursuant to Article 21 of the Medical Practitioner's Act. An inquest is then performed by either a police officer or a public prosecutor to determine the cause of death from the circumstances. If criminality is established, a judicial autopsy is conducted according to the Criminal Procedure Act. If it is determined that criminality is not involved, an administrative autopsy is performed according to the Criminal Procedure Act by a medical coroner or a professor or associate professor of the department of forensic medicine in a medical school. The forensic police officer conducts an investigation and only assists in the autopsy. The Japanese coroner

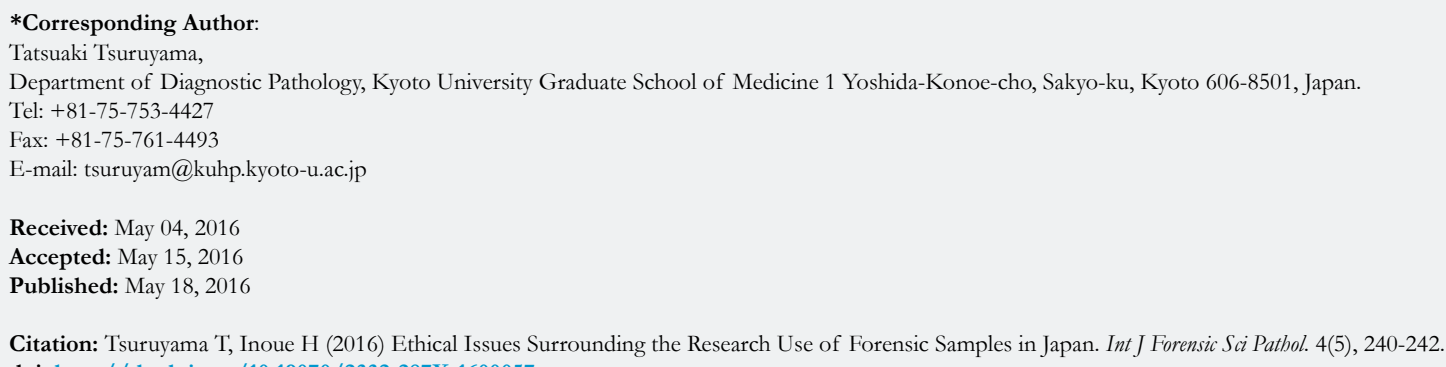

Copyright: Tsuruyama $\mathbf{T}^{\circ}$ 2016. This is an open-access article distributed under the terms of the Creative Commons Attribution License, which permits unrestricted use, distribution and reproduction in any medium, provided the original author and source are credited. 
system was introduced after World War II.

In UK, an appointed government official conducts all of the procedures involving the investigation and autopsy. In the United States, qualifications for coroners are established by individual states. Thus, the qualifications for coroners vary widely.

\section{Administrative Autopsies without IC}

Administrative autopsies are stipulated under the Autopsy Preservation Act. In addition to this law, with respect to the Food Sanitation Act, an autopsy is performed as needed by an anatomist upon the order of a governor, mayor, or other official in cases where death by food poisoning is suspected (Article 28, Food Sanitation Act). According to the Quarantine Act, an autopsy is performed by a quarantine officer, by order of a quarantine station manager, in cases where death by an infectious disease is suspected (Clause 2, Article 13, Quarantine Act). Autopsies may also be performed in other cases, as necessary, with the permission of public health centers (Article 7, Autopsy Preservation Act). As a basic rule, the autopsy process does not require a relative's IC in cases where a medical coroner is present. However, the need for an autopsy is typically explained to relatives in detail, except where serious and extensive physical damage has occurred or the relatives are unknown and cannot be contacted.

In a prefecture where no medical coroner is present, the autopsy is performed by the department of forensic medicine at a medical school in the area, after obtaining IC from the relatives. In addition, the Survey of the Cause of Death and the Corpse Identification Law was enacted in 2015. As a result, the police chief can directly request a medical doctor to conduct a forensic autopsy examination after IC has been obtained from the relatives.

\section{Judicial Autopsies}

If a criminal element is possible, the public prosecutor will demand that the court issue a Written Request for an Evaluation by a Forensic Medical Expert. If the court acknowledges the need for a judicial autopsy, the judge will issue an autopsy permit. The autopsy will begin after a request for expert evidence and a written permit are presented by the police and the public prosecutor to either the medical examiner or forensic pathologist (professor or associate professor) at a medical school or university. The autopsy in this case is performed to identify the cause of death, the nature of the injury, the time that elapsed since death, and other matters pertaining to a crime-related corpse (Articles 165, 168, and 225 of the Criminal Procedure Art). This autopsy is conducted as a forced invocation of public power and does not require the relatives' consent to autopsy; although in many cases, the relatives are informed of the procedure.

\section{Discussion}

We shall investigate the conditions required for using samples obtained through forensic autopsies for research. In this article, we limit the discussion to forensic pathological research. Research use of an anatomical specimen is permitted under the Autopsy Preservation Act (Article 17 and Article 18). In 2002, the Japan Society of Forensic Pathology announced the guidelines for the forensic study of bodily fluids and organs collected and stored at a forensic facility. According to these guidelines, it is recommended that the forensic pathologist obtain approval from an institutional review board (IRB) for use of the forensic samples as well as IC from the relatives as soon as possible. In cases where it is difficult to make contact with relatives, the research use of forensic samples will only be permitted upon approval of an IRB. For the medical practitioner, the IC requirement can pose issues related to the balancing of public and private benefits [6].

Occasionally, there are discussions on forensic access to biosamples. However, there have not been extensive discussions on clinical research using these samples.

\section{Researching Endogenic Sudden Death}

We will first consider a case in which we investigate endogenic sudden death using forensic samples. At present, sudden death is defined by the World Health Organization (WHO) as death that occurs within 24 hours of the onset of a disease. In Japan, approximately $10 \%$ of deaths are believed to be endogenic sudden deaths. According to a report by the Tokyo Medical Examiners' Office, two-thirds of all endogenic sudden deaths are caused by acute coronary syndrome - ischemic heart diseases due to lethal arrhythmia (http://www.fukushihoken.metro. tokyo.jp/english/index.html) [7,8]. Forensic samples are used to analyze the pathology of the cause of death attributable to acute changes in adults and children. Pediatric cases present unique issues such as sudden infant death syndrome $[9,10]$. A recent survey of public attitudes regarding the use of residual newborn screening specimens indicates a supportive response to research use for forensic studies. Ethical issues arise in using samples for research purposes after obtaining approval from an IRB [11]. For example, for autopsies based on the Quarantine Act, we can analyze common forensic pathological data and utilize the data to prevent similar infections in the future. Likewise, there may be cases where research use is permitted from a crime prevention perspective and we determine that there are few ethical reviews of the research that was used to investigate a cause of the death where the study is compatible with the purpose of the autopsy.

Further, let us examine whether it is possible to use samples for the research of chronic diseases that are not causes of endogenic sudden death. In analyzing autopsy cases, it is actually difficult to distinguish an acute change in endogenic sudden death from a chronic change. Although an investigation of chronic histologic change leads to a better understanding of an acute change, we found that there are few studies that use samples to investigate endogenic sudden death following a long-term course such as chronic myocardial infarction.

\section{Use of Samples as a Reference in Clinical Studies}

Lastly, we examine cases where IC was not obtained for an autopsy and whether it is possible to use the forensic samples collected as a disease reference sample rather than for analyzing the forensic pathology. Because normal samples are more difficult to obtain in a pathological autopsy than through surgical or biopsy samples, the need may arise to use trauma-related forensic samples as normal disease samples.

The court adjudicates cases for judicial autopsy based on public health/hygiene or judicial/administrative factors. The forensic 
medical laboratory in a medical college or university manages forensic samples on behalf of or on consignment by the court in light of the legislative intent of the Autopsy Preservation Act that discourages impairing the dignity of forensic sample donors. For this reason, we must ensure that the use of forensic samples as normal control samples rigorously complies with EGM. In addition, grounds for justifying the research use of forensic samples must be clearly shown in the content of the research program that is approved by the IRB.

\section{Scientific Justification for Forensic Sample Use}

Presently in the EGM, the research use of forensic samples is permitted when the IRB approves a research program after considering the ethical issues [13]. In UK, according to Part 1 of the Human Tissue Act (HTAct) 2004, IC is a condition for research use of autopsy specimen, unless (a) it has imported, nor (b) it has come from those who died before at least 100 years before the act comes into force [14]. Both Swiss and UK regulations, in principle, impose obtaining IC from the person before his death, or from his relatives or the designated person.

Herein, we propose that the research use of forensic samples without IC is permitted in any of the following cases: (i) when the use is critical to research; (ii) when it is difficult to substitute the use of a forensic sample with the use of other pathological samples; or (iii) when the use is justifiable from a scientific perspective, such as feasibility.

\section{Conclusion}

We investigated the research use of forensic samples that were obtained without IC from a patient's relatives. If samples are to be used, an IRB approve the research use on the basis of a detailed research program based on scientific perspectives and in accordance with the guidelines.

\section{References}

[1]. Inoue H, Tsuruyama T. (2015) Issues concerning clinical research using human pathologic bioresources in the biobank era. Int J of Forensic Sci Pathol 3(10): 176-178.

[2]. Inoue H, Tsuruyama T. (2016) Regulations of regenerative medicine including clinical application of iPS cells in Japan. Int J Stem Cell Res Transplant 4(4): 179-181.

[3]. Japanese Ministry of Education, Culture, Sports, Science and Technology MEXT, Life Portal site http://www.lifescience.mext.go.jp/files/pdf/ n1500_01.pdf.

[4]. Schraeder PL, Delin K, McClelland RL, So EL. (2009) A nationwide survey of the extent of autopsy in sudden unexplained death in epilepsy.Am J Forensic Med Pathol. 30(2):123-126.

[5]. Yoshioka H, Tsuji C, Kajii E, Suzuki H, Tanaka N, Kato H, Utsugi S, et al. (2002) Use in the forensic study of the organs and body fluids that have been collected and stored in the forensic medical and other areas. Japanese Legal Med J 56(2 \& 3): 319-322.

[6]. Ranson D. (2011) Sexual assault examinations and forensic medical samples. J Law Med 19(1): 32-35.

[7]. Kakimoto Y, Tsuruyama T, Miyao M, Abiru H, Sumiyoshi S, et al. (2013) The effectiveness and limitations of triphenyltetrazolium chloride to detect acute myocardial infarction at forensic autopsy. Am J Forensic Med Pathol 34(3): 242-247.

[8]. Kakimoto Y, Ito S, Abiru H, Kotani H, Ozeki M, et al. (2013) Sorbin and $\mathrm{SH} 3$ domain-containing protein 2 is released from infarcted heart in the very early phase: Proteomic analysis of cardiac tissues from patients. J Am Heart Assoc 2: e000565.

[9]. Odendaal HJ, Elliott A, Kinney HC, Human M, Gaspar D, et al. (2011) Consent for autopsy research for unexpected death in early life. Obstet Gynecol. 117(1):167-71.

[10]. Weber MA, Epstein J, Simons J, Malone M, Ashworth M, Sebire NJ. (2007) Challenges to implementation of the new Coroners' (Amendment) Rules 2005: experience from a tertiary paediatric pathology centre. Med Sci Law. 47: 293-8.

[11]. Botkin JR, Rothwell E, Anderson R, Stark L, Goldenberg A, Lewis M, Burbank M, Wong B. (2012) Public attitudes regarding the use of residual newborn screening specimens for research. Pediatrics 129: 231-238.

[12]. Elger BS, Hofner MC, Mangin P (2009) Research involving biological material from forensic autopsies: legal and ethical issues. Pathobiology 76(1): $1-10$.

[13]. The Federal Assembly of the Swiss Confederation. (2011) Federal Act on Research involving Human Beings (Human Research Act, HRA) of 30 September 2011 (Status as of 1 January 2014). https://www.admin.ch/opc/en/ classified-compilation/20061313/index.html

[14]. (UK) Human Tissue Act 2004 CHAPTER 30 http://www.legislation.gov. uk/ukpga/2004/30/introduction 